\title{
Comparison and Combination of Energy and Wavelength Dispersive X-Ray Spectrometry in Electron Probe Microanalysis of Minerals and Glasses
}

\author{
Karsten Goemann ${ }^{1}$
}

1. Central Science Laboratory, University of Tasmania, Hobart TAS 7001, Australia

Standards-based quantitative energy dispersive x-ray spectrometry (EDS) has been in use for over 40 years (e.g. [1]). Modern EDS systems on scanning electron microscopes (SEM) provide excellent throughput and long-term stability. At University of Tasmania, Kakanui Hornblende [2] was measured as a secondary standard by multiple users in 77 sessions over 5 years on a Hitachi SU-70 Schottky field emission SEM with Oxford AZtec XMax $80 \mathrm{~mm}^{2}$ EDS. This method was designed for speed to determine internal standards for laser ablation ICP-MS and ultimate accuracy was not required. The conditions were $15 \mathrm{kV}$ accelerating voltage, 2-3 nA beam current, beam scan area $\approx 10 \mu \mathrm{m}, 5 \mathrm{~s}$ live time, dead time 40-50\%, and typically $35 \mathrm{kcps}$ throughput at $133 \mathrm{eV} \mathrm{Mn} \mathrm{K \alpha}$ spectral resolution. An indirect beam current measurement was performed for each session on Co metal. In spite of only occasional energy and element re-calibration, the long-term standard deviation (s.d.) for all elements except $\mathrm{O}$ and $\mathrm{Si}$ is well within the (single point) precision of the measurement, see Fig. 1.

In spite of findings that EDS can match or even exceed accuracy and precision of wavelength dispersive X-ray spectrometry (WDS) $[3,4]$, many still consider WDS on an electron microprobe (EPMA) the gold standard for quantitative microanalysis. For direct comparison, a 195-point grid was acquired simultaneously for EDS and WDS on the USGS basaltic glass standard BHVO-2G on a JEOL JXA8530F Plus EPMA with five WDS and a Thermo UltraDry Extreme 30mm EDS at $15 \mathrm{kV}, 20 \mathrm{nA}$, and $10 \mu \mathrm{m}$ beam diameter, using the large EDS aperture and $4.0 \mu \mathrm{s}$ time constant for $\approx 40 \mathrm{kcps}$ stored and $\approx 38 \%$ dead time. EDS counting of $90 \mathrm{~s}$ live time was matched to the total WDS counting times. Quantification for both EDS and WDS was performed in Probe Software's Probe For EPMA package, using the same primary standards and matrix corrections, conventional WDS off-peak backgrounds, and the top hat filter fit in Thermo Pathfinder to generate EDS net intensities. For all elements $>1 \mathrm{wt} \%$, accuracy of the two techniques is similar, see Table 1. For the lower level elements K, Mn, and P, accuracy by WDS is significantly better. This can potentially be improved by EDS processing optimisation. S.d. of the 195 points is lower for EDS for all elements except Ti, P, and especially Mn.

To make the most of the techniques' strengths (stability and flexibility for EDS, sensitivity and spectral resolution for WDS) they can be combined on modern EPMA instruments for simultaneous acquisition of a full ED spectrum for major and minor elements and selected trace elements on the several WDS. This has been used on the same EPMA as above for point measurements on minerals like rutile (Ti on EDS; Si, Ca, V, Cr, Mn, Fe, Zr, Nb, Sn, Ta, W on WDS; 20 kV, 200 nA, small EDS aperture) or titanite ( $\mathrm{Si}, \mathrm{Ca}$, Ti on EDS; Na, Mg, Al, V, Cr, Mn, Fe, Sr, Y, Zr, Nb, selected REE on WDS). As an added benefit, any of the WDS elements can also be quantified afterwards using the corresponding ED spectra, see the example in Fig. 2. For Fe the agreement is excellent for the whole range of 0.1-0.9 wt\%, indicating that Fe does not have to be measured by WDS in this case. For Nb there are several data points with substantially higher values by EDS. Investigating the corresponding ED and WD spectra reveals that these grains contain substantial Mo which was initially not included in the method and appears to create interference issues in EDS. Further examples including for mapping will be presented. 


\section{References:}

[1] SJB Reed, NG Ware, J. Petrol. 16 (1975), p. 499.

[2] E Jarosewich, JA Nelen and JA Norberg, Geostand. Newslett. 4 (1980), p. 43.

[3] NWM Ritchie, DE Newbury, JM Davis, Microsc. Microanal. 18 (2012), p. 892.

[4] DE Newbury, NWM Ritchie, J. Mater. Sci. 50 (2015), p. 493.

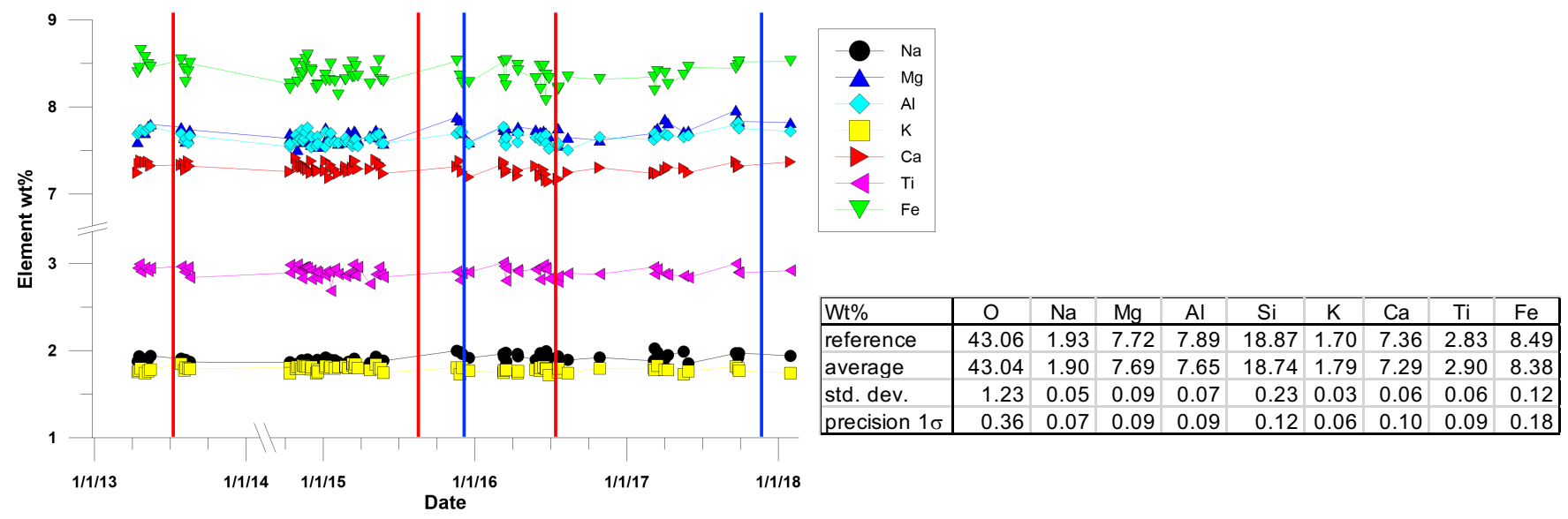

Figure 1. Kakanui Hornblende over time by SEM-EDS. Each data point represents an average of 3 measurements. Red vertical lines indicate energy calibration, blue lines re-calibration of the elements.

\begin{tabular}{|c|c|c|c|c|c|c|c|c|c|c|}
\hline BHVO-2G, $\mathrm{n}=195$ & $\mathrm{Si}$ & $\mathrm{Ti}$ & $\mathrm{Al}$ & $\mathrm{Fe}$ & $\mathrm{Mn}$ & $\mathrm{Mg}$ & $\mathrm{Ca}$ & $\mathrm{Na}$ & $\mathrm{K}$ & $\mathrm{P}$ \\
\hline Reference, wt $\%$ & 23.30 & 1.63 & 7.16 & 8.63 & 0.129 & 4.36 & 8.17 & 1.64 & 0.43 & 0.120 \\
\hline \multicolumn{11}{|l|}{ WDS } \\
\hline Xtal & TAP & PETL & TAP & LiFL & LiFL & TAPL & PETL & TAPL & PETL & PETL \\
\hline Counting, seconds & 20 & 30 & 20 & 20 & 30 & 20 & 20 & 20 & 20 & 20 \\
\hline Intensity, cps/nA & 477 & 52 & 129 & 59 & 1.4 & 181 & 257 & 33 & 10.8 & 0.8 \\
\hline Average wt\% & 23.45 & 1.63 & 7.25 & 8.47 & 0.131 & 4.34 & 8.05 & 1.61 & 0.42 & 0.115 \\
\hline Std. dev., wt $\%$ & 0.11 & 0.010 & 0.04 & 0.13 & 0.010 & 0.02 & 0.05 & 0.03 & 0.014 & 0.009 \\
\hline Precision $1 \mathrm{~s}, \mathrm{wt} \%$ & 0.05 & 0.010 & 0.03 & 0.06 & 0.009 & 0.02 & 0.03 & 0.01 & 0.008 & 0.009 \\
\hline \multicolumn{11}{|l|}{ EDS (90 s live) } \\
\hline Intensity, cps/nA & 558 & 17 & 168 & 42 & 1.3 & 96 & 113 & 30 & 6.0 & 3.2 \\
\hline Average wt\% & 23.45 & 1.66 & 7.13 & 8.43 & 0.213 & 4.26 & 8.04 & 1.65 & 0.38 & 0.159 \\
\hline Std. dev., wt\% & 0.06 & 0.02 & 0.03 & 0.09 & 0.05 & 0.02 & 0.03 & 0.02 & 0.01 & 0.01 \\
\hline Precision 1s, wt $\%$ & 0.08 & 0.03 & 0.03 & 0.08 & 0.02 & 0.02 & 0.04 & 0.02 & 0.02 & 0.01 \\
\hline
\end{tabular}

Table 1. Comparison of simultaneous EDS and WDS EPMA measurements performed on BHVO-2G.
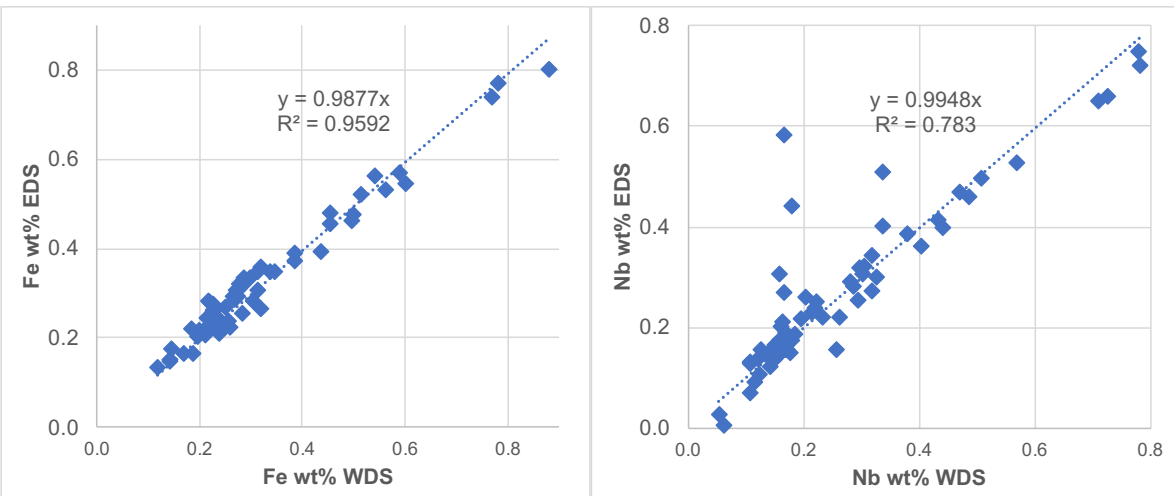

Figure 2. Comparison of $\mathrm{Fe}$ and $\mathrm{Nb}$ by simultaneous EDS and WDS in natural rutile crystals. 